therapy, with minimal and questionable benefit. Subsequent DNA analyses on blood showed homozygous deletion mutation in exons 7 and 8 of the survival motor neuron $(\mathrm{SMN})$ gene. The final molecular diagnosis was SMA type I. One patient died at 5 months of age of pneumonia and respiratory failure, and the other was currently surviving on a respirator. (Ravid S, Topper L, Eviatar L. Acute onset of infantile spinal muscular atrophy. Pediatr Neurol May 2001;24:371-372). (Dr Eviatar, Division of Pediatric Neurology, Schneider's Children Hospital, New Hyde Park, NY 11040).

COMMENT. Several atypical presentations of infantile spinal muscular atrophy are cited from the literature. The acute onset of weakness and areflexia following a respiratory infection suggested a Guillain Barre syndrome, and the NCS were confirmatory. The very young age, less than 6 months, and the absent response to immunoglobulin were against this diagnosis. SMA type I was established by DNA molecular analyses. The final outcome with respiratory failure was expected.

\title{
FACIAL ELECTROMYOGRAPHY IN NEONATAL FACIAL PALSY
}

Electromyography (EMG) studies of 172 children with congenital facial palsy were analysed retrospectively at the Hopital d'Enfants Armand-Trousseau, Paris, France. The first clinical and EMG exams were done from 3 to 90 days after birth in 103 infants, 3 to 12 months in 49, and from 1 to 2 years in 20 children. In 85 with unilateral congenital facial paralysis without malformation, 48 were attributed to perinatal forceps or other trauma. Clinical and EMG monitoring showed slow nerve regeneration and muscular reinervation even after 2 years. Asymmetrical crying facies occurred in 51 infants, the EMG showed partial denervation of the depressor anguli oris and depressor labia inferioris, and a diagnosis of partial facial palsy with only the lower lip involved. Orofacial malformations with asymmetrical facial motility were studied in 33 infants. EMG activity was either absent (10 infants), neurogenic (9), or myopathic (14). Pseudoparalysis asymmetry of postural origin occurred in 5 of 172 cases of congenital facial palsy. The EMG was normal and the functional asymmetry resolved within 2 months. Mobius syndrome in 22 infants had heterogeneous EMG findings with predominance of axonal loss. Congenital facial diplegia with muscular diseases, eg myotonic dystrophy, congenital muscular dystrophy, metabolic myopathies, facioscapulohumeral dystrophy with early onset facial weakness. In Orofacial apraxia with cerebral palsy, EMG of facial muscles is normal, except when brain stem nuclei are involved. In 12 infants with dysphagia at birth and failure to thrive, a neurogenic EMG indicated lesions of VII to XII cranial nerves. (Renault F. Facial electromyography in newborn and young infants with congenital facial weakness. Dev Med Child Neurol June 2001;43:421427). (Francis Renault MD, Unite de Neurophysiologie Clinique, Hopital d'Enfants ArmandTrousseau, 26 avenue du Docteur Arnold-Netter, 75571 Paris 12, France).

COMMENT. The author advocates EMG of facial muscles in infants to differentiate causes of congenital facial palsy and to evaluate course and prognosis. Electrodiagnostic studies of Mobius syndrome may shed light on the pathophysiology. The author provides details regarding his technique of EMG and findings, and values for motor latencies of facial muscles and facial nerve conduction velocities from birth $(19 \mathrm{~m} / \mathrm{s})$ to 3 years $(39 \mathrm{~m} / \mathrm{s})$. 